\title{
Ortorombik metaborik asit molekülünün moleküler yapısı ve elektronik özellikleri üzerindeki konformasyonel etkinin teorik olarak incelenmesi
}

\section{Güventürk Uğurlu*}

Kafkas Üniversitesi, Fen-Edebiyat Fakültesi, Fizik Bölümü, 36100 Kars, Türkiye, ORCID ID orcid.org/0000-0003-4171-7879

\section{MAKALE BILGISi \\ Makale geçmişi: \\ İlk gönderi 27 Aralık 2019 \\ Revize gönderi 01 Mayıs 2020 \\ Kabul 02 Haziran 2020 \\ Online 29 Haziran 2020}

\section{Araştırma Makalesi}

DOI: 10.30728/boron.666064

Anahtar kelimeler:

B3LYP,

$\mathrm{C}_{3 \mathrm{~h}}$,

Konformasyon analizi,

NLO,

Ortorombik metaborik asit.

\begin{abstract}
ÖZET
Bu çalışmada, ortorombik metaborik asit molekülünün konformasyon analizi, doğrusal olmayan optik davranışı, titreşim spektrumları, elektronik ve moleküler yapısı ab initio Hartree Fock (HF) ve Yoğunluk Fonksiyonel Teorisi DFT/B3LYP temel seviyesinde $6-311++G(d, p)$ temel seti kullanılarak kapsamlı bir şekilde araştırıldı. Konformasyon analizi hem $\varphi(\mathrm{B} 1-\mathrm{O} 1-\mathrm{H})$ bağ açısı, hem de $\psi(\mathrm{O} 4-\mathrm{B} 1-\mathrm{O} 1-\mathrm{H})$ dihedral açısının fonksiyonu olarak ilk kez detaylı olarak yapıldı. Hesaplanan potansiyel enerji eğrilerinin sonuçları, molekülün minimum enerjili iki kararlı konformere (C-I ve C-Il konformer) sahip olduğunu gösterdi. C-I konformeri C-II konformerinden daha kararlıdır. Ortorombik metaborik asit molekülünün C-I ve C-II konformerlerinin doğrusal ve doğrusal olmayan optik özellikleri, elektrik dipol momenti $\mu$, polarizebilite $\alpha$ ve hiperpolarizebilitesi $\beta$ her iki yöntem ile incelenmiştir. Molekülün $\mathrm{C}$-I ve $\mathrm{C}$-II konformerlerinin optimize edilmiş moleküler yapıları, sırasıyla, $C_{3 h}$ ve $C_{s}$ simetrisine sahiptir. $C_{s}$ simetrisine sahip $C$-II konformeri için B3LYP/6-311++G(d,p) ve HF/6-311++G(d,p) yöntemleri kullanılarak elde edilen dipol momenti değerleri 2,95 ve 3,07 Debyedir. Oysa $C_{3 h}$ simetrisine sahip C-I konformeri için aynı yöntemler kullanılarak elde edilen değerlerin eşit olduğu $(0,0$ Debye) bulunmuştur. Her iki konformerin titreşim modlarının işaretlenmesini bulmak için toplam enerji dağılımı (TED) VEDA 4f programı kullanılarak hesaplanmıştır. Literatürdeki deneysel veriler ile hesaplanan yapısal parametreler arasında iyi bir uyum olduğu görülmüştür.
\end{abstract}

\section{Theoretical examination of the conformational effect on the molecular structure and electronic properties of the orthorhombic metaboric acid molecule}

\section{ARTICLE INFO}

\section{Article history:}

Received 27 December 2019

Received in revised form 01 May 2020

Accepted 02 June 2020

Available online 29 June 2020

Research Article

DOI: 10.30728/boron.666064

Keywords:

B3LYP,

$\mathrm{C}_{3 \mathrm{~h}}$

Conformational analysis,

NLO,

Orthorhombic metaboric acid.

\begin{abstract}
In the present study, conformational analysis, Nonlinear Optical (NLO) behavior, vibrational spectra, electronic and the molecular structure of orthorhombic metaboric acid have been investigated comprehensively by using ab initio Hartree Fock (HF) and Density Functional Theory DFT at the B3LYP level with 6-311++G(d,p) basis set. The conformational analysis was performed in detail for the first time as a function of both the $\varphi(\mathrm{B} 1-\mathrm{O} 1-\mathrm{H})$ bond angle and the $\psi(\mathrm{O} 6-\mathrm{B} 1-\mathrm{O} 1-\mathrm{H})$ dihedral angle. The results of calculated potential energy curves show that the molecule has two conformers (C-I and C-Il Conformer) with minimum energies in a stable form. C-I conformer is a more stable form than C-II conformer. Linear and nonlinear optical properties of conformer $\mathrm{C}-\mathrm{I}$ and $\mathrm{C}-\mathrm{II}$ of the orthorhombic metaboric acid molecule are examined by the determination of the electric dipole moment $\mu$, the polarizability $\alpha$, and the hyperpolarizability $\beta$ both methods. The optimized molecular structures of $\mathrm{C}-\mathrm{I}$ and $\mathrm{C}$-II conformer of the molecule belong to $\mathrm{C}_{3 \mathrm{~h}}$ and $\mathrm{C}_{\mathrm{s}}$ symmetry, respectively. The dipole moment values for conformer $\mathrm{C}$-II with $\mathrm{C}_{\mathrm{s}}$ symmetry obtained using B3LYP/6$311++G(d, p)$ and $H F / 6-311++G(d, p)$ methods were found to be 2.95 and 3.07 Debye. Whereas, for $\mathrm{C}-\mathrm{I}$ conformer with $\mathrm{C} 3 \mathrm{~h}$ symmetry, the values obtained using the same methods are found equal (0.0 Debye). Total energy distributions (TED) were calculated to find assignments of calculated vibration modes of both conformers by using VEDA $4 \mathrm{f}$ program. It was observed that there is a good agreement between the experimental data in the literature and the calculated structural parameters.
\end{abstract}




\section{Giriş (Introduction)}

Bor atomu yaptığı kovalent bağlı bileşiklerinde, valans orbitallerinin $\mathrm{sp}^{2}$ den $\mathrm{sp}^{3}{ }^{3} \mathrm{e}$ hibritlenmesi sonucu üç elektronla katkıda bulunması ve elektron çifti alıclsı olmasından dolayı dört bağ yapma eğilimine sahip önemli bir elementtir $[1,2]$. Bor doğada saf halde bulunmayıp, genelde borik asit ve borun diğer bileşikleri halinde bulunur $[3,4]$. Bu bileşiklerin büyük kısmını borik asit ve borun sodyum, magnezyum ve kalsiyum ile oluşturduğu bileşiklerdir. Literatürde, hidrojen borat ve ortoborik asit olarak da bilinen borik asit $\left[\mathrm{H}_{3} \mathrm{BO}_{3}\right.$ veya $\left.\mathrm{B}(\mathrm{OH})_{3}\right)$ ] molekülü; tekstil, kozmetik, cam sanayi gibi önemli alanlarda kullanılmaktadır [5]. Bor kayalarda, toprakta ve suda bulunması ile birlikte toprakta yetişen bitkilerin hücre duvarlarında az miktarda olsa bulunduğundan doğada hemen hemen her yerde bulunur $[6,7]$. Bor içerikli bileşikler yüzyıldan fazla bir süredir antiseptikler, antibiyotikler, kozmetikler ve böcek öldürücüler olarak kullanılmaktadır $[8,9]$. Son yıllarda, bu bileşiklerin antibiyotikler ve antikanser ilaçları olarak kullanım alanları genişlemektedir [10,11]. Ayrıca, borik asitli bileşikler son zamanlarda özellikle tıptan ilaçlamaya, endüstriyel ürünlerde birçok alanda kullanımından ve terapötik etkilerinden dolayı araştırmacıların dikkatini çekmeye devam etmektedir. İlaçlama alanında, dünya genelinde yaşamı tehdit eden sıtma, dang ve zika hastalıkların yayılmasında vektör olan Aedes aegypti (L.) and Aedes albopictus (Skuse) [12-14] gibi sivrisinek türlerinin kontrol altında tutulmasında kullanılan etkin madde borik asittir $[15,16]$. Boroksinler de borik asitler gibi endüstriyel sanayi üretimi ve uygulamalarda farklı alanlarda kullanılan önemli bileşiklerdir. Bunlardan bazıları, alev geciktirici olarak yanıcı malzemelerin, daha geç tutuşmalarını sağlamak için katkı maddesi olarak kullanılıyor [17], bazıları ise kaynak ve lehim işlemlerinde katalizör [18] ve lityum iyon pillerde elektrolit katkı maddesidir [19]. Su-bor oksit sistemindeki sıvıda oluşan kristal fazın soğutulduklarında ortoborik asit, üç farklı modifikasyonlu meta-borik asit ve bor oksit kristalleri şeklinde olduğu bilinmektedir [20,21]. Şöyle $\mathrm{ki}$, normal sıcaklıklarda kararlı bir yapıya sahip olan borik asit, ergime noktasına ısıtıldığı zaman, çeşitli fiziksel ve kimyasal reaksiyonlar meydana gelir. 100 ${ }^{\circ} \mathrm{C}$ 'nin üzerinde borik asitte biriken nem buharlaşır.
Sıcaklık daha da arttıkça, borik asit dehidratlaşıyor, böylece borik asidin dehidratlaşma süreci iki basamaklı reaksiyonla oluşur $[20,22]$.

$$
\begin{aligned}
2 \mathrm{H}_{3} \mathrm{BO}_{3} & \longrightarrow 2 \mathrm{HBO}_{2}+2 \mathrm{H}_{2} \mathrm{O} \\
2 \mathrm{HBO}_{2} & \longrightarrow \mathrm{B}_{2} \mathrm{O}_{3}+\mathrm{H}_{2} \mathrm{O}
\end{aligned}
$$

Dehidratlaşmanın birinci basamağında borik asit molekülü sıcaklığa bağlı (105-160 C sıcaklık aralığında) olarak meta borik asidin üç farklı kristal modifikasyonuna dönüşür. Bu üç farklı kristal modifikasyon; ortorombik metaborik asit $\left(\alpha-\mathrm{HBO}_{2}-\mathrm{III}\right), \mathrm{HBO}_{2}-\mathrm{III}, 130{ }^{\circ} \mathrm{C}$ civarında; monoklinik metaborik asit $\left(\beta-\mathrm{HBO}_{2}\right), \mathrm{HBO}_{2}-\mathrm{II}, 130-150$ ${ }^{\circ} \mathrm{C}$ aralığında; kübik metaborik asit $\left(\mathrm{\gamma}-\mathrm{HBO}_{2}\right), \mathrm{HBO}_{2}-\mathrm{I}$, $150-160^{\circ} \mathrm{C}$ sıcaklık aralığında oluşmaktadır. Çalışılan molekülle ilgili yapılan literatür araştırmalarında, ortorombik metaborik asit [23], monoklinik metaborik asit [24] ve kübik metaborik asit [25] moleküllerinin yapıları deneysel olarak aydınlatılığı görülmektedir. Metaborik asit ve türevlerinin moleküler özellikleri ile titreşim spekturumları deneysel ve teorik çalışılmasına [26-28] rağmen molekülün konformasyon analizi, elektronik ve doğrusal olmayan optik özelliklerinin deneysel ve teorik olarak çalışılmadığı görülmüştür. Bu çalışmada, ortorombik metaborik asit molekülünün potansiyel enerji eğrileri taranarak detaylı bir konformasyon analizi yapıldı ve molekülün iki kararlı konformeri belirlendi. Her iki konformerin yapısal parametreleri, titreşim frekansları, $\mu, \alpha, \beta, E_{\text {Hомо }}, E_{\text {LUMO }}$ ve $\Delta E g=E_{\text {LUMо }}-E_{\text {Hомо }}$ değerleri DFT/B3LYP ve HF metodları ile farklı taban setleri kullanılarak hesaplanmıştır. Hesaplamalar sonucunda, bu iki konformerin farklı kimyasal ve fiziksel özelliklere sahip olduğu görüldü. Belirlenen bu önemli moleküler özelliklerin ortorombik metaborik asit molekülü ile yapılacak yeni çalışmalara yardımcı olacağı ve molekülün kullanım alanlarını genişleteceği düşünülmektedir. Ortorombik metaborik asit molekülünün deneysel olarak belirlenen kristal fazındaki [29] yapısı ve bu çalışmada teorik olarak B3LYP/6-311++G(d,p) yöntemi ile C-I konformerinin optimize edilmiş ve numaralandırılmış kimyasal yapısı sırasıyla Şekil 1(a) ve (b)'de verilmiştir.
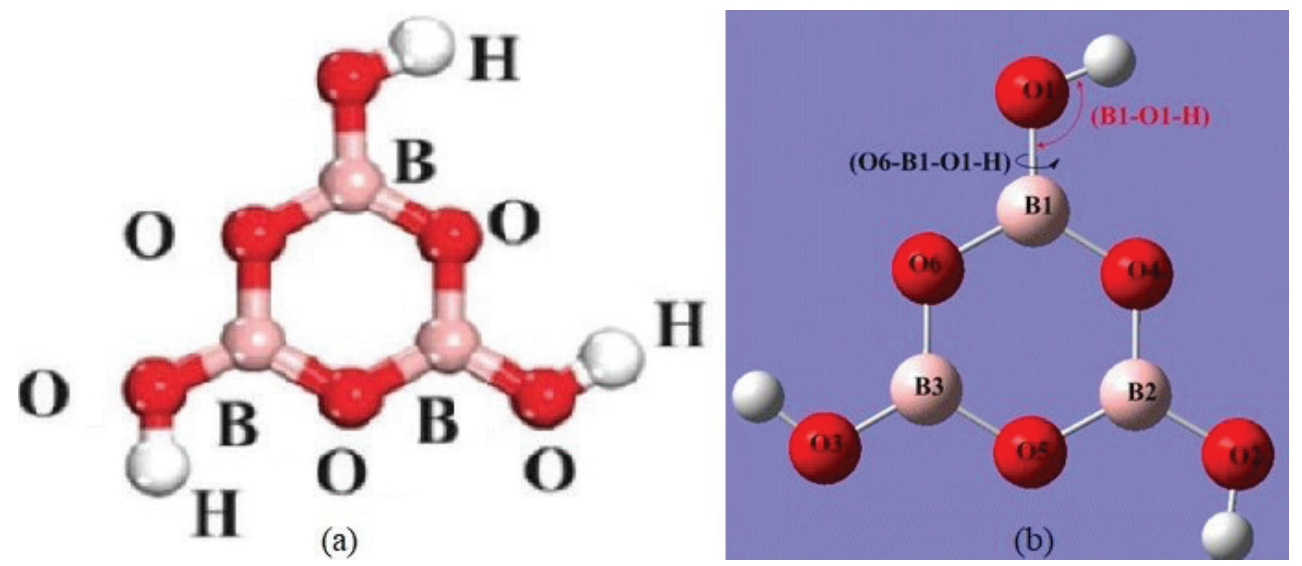

Şekil1. (a) kristal fazındaki (b) C-II konformerinin numaralandırılmış moleküler yapısı ((a) In crystal phase (b) The molecular structure of C-Il conformer showing the atomic numbering). 


\section{Malzeme ve yöntem (Material and method)}

Bu çalışmada, ilk olarak ortorombik metaborik asit molekülünün gaz fazında üç boyuttaki yaklaşık geometrisi GaussView [30] programında çizilerek GAUSSIAN 09 paket programında [31] giriş verisi olarak kullanıldı ve konformasyon analizi yoğunluk fonksiyoneli DFT/ Becke'nin 3 parametreli hibrit değiş-tokuş fonksiyoneli (B3) [32] ile Lee-Yang ve Parr'ın korelasyon fonksiyonelinden $[33,34]$ oluşan B3LYP teori seviyesinde $6-311++G(d, p)$ taban seti kullanılarak konformasyonel dağılım hesaplamaları, ilgili parametrelerin ( $\varphi$ (B1$\mathrm{O} 1-\mathrm{H})$ ve $\Psi$ (O4-B1-O1-H)) dışında hiçbir sınırlama olmaksızın yapıldı. Hesaplamalarda, ortorombik metaborik asit molekülünün konformasyon analizi yapılarak hem $\varphi(\mathrm{B} 1-\mathrm{O} 1-\mathrm{H})$ bağ açısının hem de $\Psi$ (O4B1-O1-H) dihedral açının fonksiyonu olarak potansiyel enerji eğrileri hesaplatılmıştır. Konformasyon analizi sonucunda elde edilen düşük enerjili iki "minimum" elde edilmiştir. Konformasyonların adlandırılması, molekülün B-O-H bağ açılarını oluşturan $\mathrm{B}-\mathrm{O}$ ve $\mathrm{O}-\mathrm{H}$ bağlarına göre tanımlanmıştır. Eğer $\mathrm{O}-\mathrm{H}$ bağları $\mathrm{B}-\mathrm{O}$ bağları doğrultusunun sağına yönelmiş ise (saat yönünde) konformer C-I, O-H bağlarından biri bu yönelimlerin tersi durumda ise (saat yönünün tersi yönünde) konformer C-II olarak belirlenmiştir.

Konformasyon analizi sonucu elde edilen potansiyel enerji eğrileri üzerindeki düşük enerjili konformasyonları DFT/B3LYP ve Hartree Fock (HF) metotları ile $6-311++G(d, p)$ taban seti kullanılarak optimize edilmiştir [35]. Optimize sonucunda molekülün kararlı yapıları olan C-I ve C-II konformerleri oluşturuldu. Ortorombik metaborik asit molekülünün konformer C-I ve konformer C-Il her iki modelde titreşim frekansları dipol moment $(\mu)$, polarizebilite $(\alpha)$, hiperpolarizebilite $(\beta)$, en yüksek dolu molekül orbital (HOMO), en düşük boş molekül orbital (LUMO) değerleri hesaplanmıştır. Hesaplamalar sonucunda $\alpha$ ve $\beta$ değerlerinin kartezyen bileşenleri (a.u) belirlenmiştir. Kartezyen bileşen değerlerinden polarizebilite;

$\alpha=\left(\frac{1}{3}\right)\left(\alpha_{x x}+\alpha_{y y}+\alpha_{z z}\right)$

hiperpolarizebilite;

$\beta=\left[\left(\beta_{x x x}+\beta_{x y y}+\beta_{x z z}\right)^{2}+\left(\beta_{y y y}+\beta_{y z z}+\beta_{y x x}\right)^{2}+\left(\beta_{z z z}+\beta_{z x x}+\beta_{z y y}\right)^{2}\right]^{1 / 2}$

Eşitlikleri ile hesaplandı. Hesaplanan HOMO- LUMO enerji değerlerinden eşitlik yardımı ile molekülün enerji aralığı $\Delta$ Eg elde edildi.

$\Delta \mathrm{E}_{\mathrm{g}}=\mathrm{E}_{\mathrm{LUMO}}-\mathrm{E}_{\text {HOMO }}$

Ayrıca, ortorombik metaborik asit molekülünün, B3LYP/6-311G++(d,p) ve HF/6-311G++(d,p) teori seviyesinde, moleküler elektrostatik potansiyel (MEP) haritası hesaplanmıştır.

\section{Sonuçlar ve tartışma (Results and discussion)}

\subsection{Konformasyonel analiz (Conformational analysis)}

Bir molekülün konformasyon analizi yapısal parametrelerinin (bağ uzunluğu, bağ açısı ve dihedral açısı) fonksiyonu olarak yapılabilir. Bağ açısı ve dihedral açılarına dayalı yapılan konformasyon analizlerinde; bağ açısı $(\varphi), 0 \leq \varphi \leq 180^{\circ}$ aralığında ve dihedral açısı $(\Psi)$ $-180 \leq \Psi \leq 180^{\circ}$ arasında bir değer alabilir. Bu çalışmada, $\varphi$ (B1-O1-H) bağ açısının bağlı yapılan konformasyon analizde; $\varphi(\mathrm{B} 1-\mathrm{O} 1-\mathrm{H})$ bağ açısının alacağı değerler B-O bağı doğrultusuna göre belirlenmiştir. $\mathrm{B}-\mathrm{O}-\mathrm{H}$ bağ açısındaki O-H grubu bağlı bulunduğu B-O bağı doğrultusunun sağ tarafında alacağı değerleri 0 ile $180^{\circ}$ aralığında sol tarafındaki bölgede ise alacağı değerleri 0 ile $-180^{\circ}$ aralığında tanımlanmıştır. Ayrıca, $\varphi(\mathrm{B} 1-\mathrm{O} 1-\mathrm{H})$ bağ açısına dayalı yapılan konformasyon analizi sonuçlarının grafiğe geçirilmesinde $-180^{\circ}$ mutlak değeri alınıp her iki grafikte minimum enerjili konformasyonlarının enerji değerleri sıfır kabul edilerek bağıl enerji grafikleri çizilmiştir. Ortorombik metaborik asit molekülünün $\varphi(\mathrm{B} 1-\mathrm{O} 1-\mathrm{H})$ bağ ve $\Psi$ (O4-B1-O1-H) dihedral açılarının fonksiyonu olarak B3LYP/6$311 \mathrm{G}++(\mathrm{d}, \mathrm{p})$ yöntemi ile hesaplanan potansiyel enerji eğrileri sırasıyla Şekil 2 ve 3'te verilmiştir. Bağ açısına bağlı konformasyon analizi $\varphi(\mathrm{B} 1-\mathrm{O} 1-\mathrm{H})$ bağ açısı $112^{\circ}$ den $-112^{\circ}$ kadar $5,5^{\circ}$ aralıklarla molekül düzleminde değiştirilerek, dihedral açının $\Psi$ (O4-B1-O1-H) fonksiyonu olarak O1-H grubu B1-O1 bağının etrafında $-180^{\circ}$ ile $180^{\circ}$ aralığında $10^{\circ}$ adımlarla değiştirilerek yapılmıştır. Molekülün her iki konformeride düzlemsel yapıda olduğu ve $\psi$ dihedral açısının fonksiyonu olarak hesaplanan potansiyel enerji eğrisinde maksimum bariyer yüksekliği $\psi$ açısının $90,0^{\circ}$ ve $270,0^{\circ}$ 'de 0,404 eV olarak hesaplanmış ve $\varphi$ bağ açısının $180,0^{\circ}$ değerinde 0,676 eV olarak bulunmuştur. $\varphi(\mathrm{B} 1-\mathrm{O} 1-\mathrm{H})$ bağ açısına dayalı elde edilen potansiyel enerji eğrisi üzerindeki tüm konformasyonlar düzlemsel yapıdadır. Ayrıca, molekülün her iki potansiyel enerji eğrileri üzerinde iki minimum bulunmakta ve bu minimumlar arasındaki enerji farkı her ikisinde aynı olup 0,0304 eV dur.

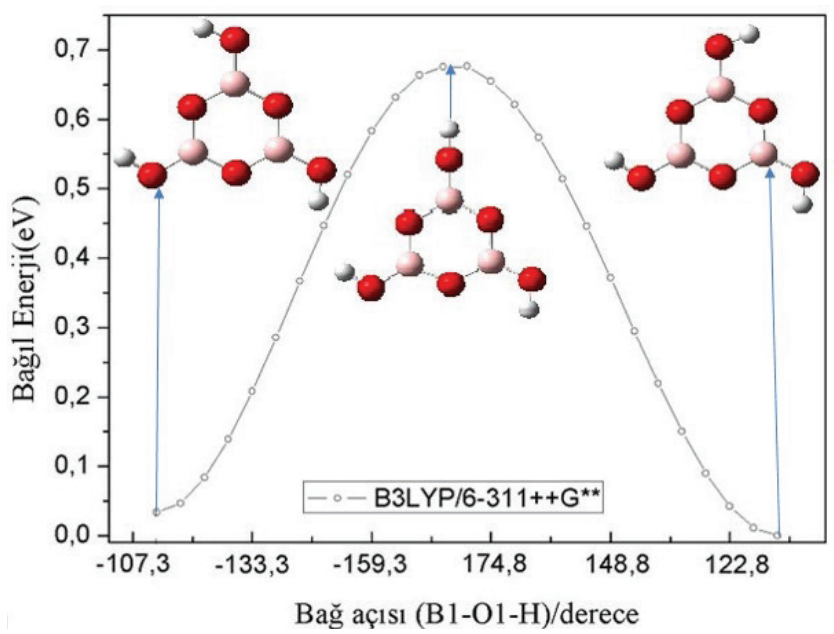

Şekil 2. $\varphi$ bağ açısının fonksiyonu olarak potansiyel enerji eğrisi (Pontential energy curve as a function of bond angle $\varphi$ ). 


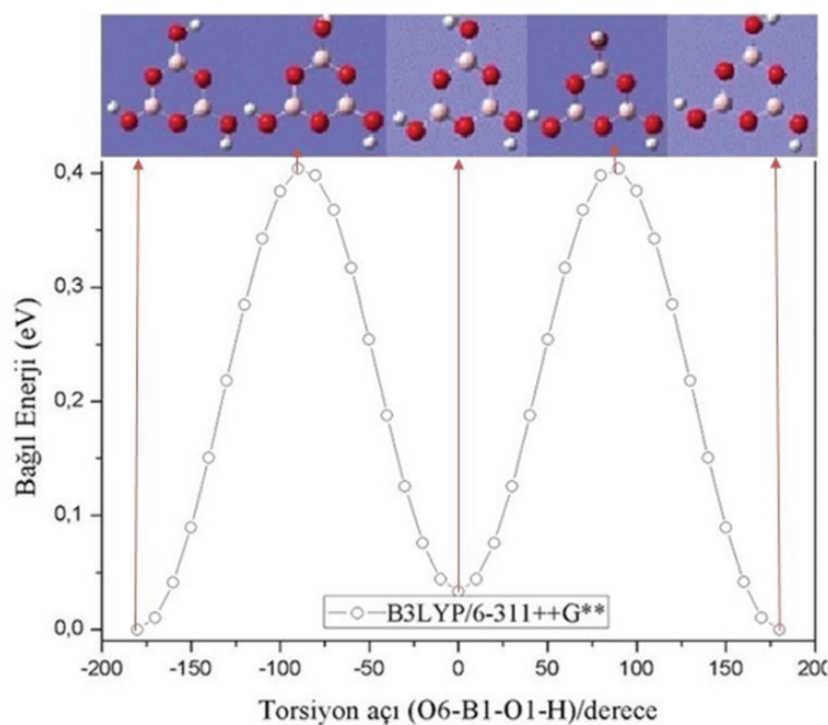

Şekil 3. $\psi$ dihedral açısının fonksiyonu olarak potansiyel enerji eğrisi (Pontential energy curve as a function of dihedral angle $\psi$ ).

\subsection{Moleküler elektrostatik potansiyel (MEP) yü- zey analizi (Analysis of molecular electrostatic poten- tial (MEP) surface)}

Moleküler elektrostatik potansiyel (MEP), moleküler bir sistemdeki atomların yükler ile birim pozitif yük arasındaki etkileşim sonucu oluşan enerji olarak tanımlanır [36]. MEP' i tanımlamada genelde renk kodlama sistemi kullanılır. Moleküler elektrostatik potansiyel yüzey haritasının üzerinde bulunan en negatif bölgeler (kırmızı bölgeler) elektrofilik atağa en yatkın, en pozitif bölgeler (mavi bölgeler) ise nükleofilik atağa en yatkın bölge olduğu şeklinde yorumlanabilinir [37]. Ayrıca, MEP haritalarının yorumlanması molekülün elektrofilik bölgeleri, nükleofilik bölgelerin belirlenmesi, hidrojen bağlarını açıklanması ve yeni kimyasalların sentezi aşamasında önemli bir faktördür [38,39]. Bu çalışma- da molekülün her iki konformeri B3LYP/6-311G++(d,p) metodu ile hesaplanan 3D MEP yüzey haritaları Şekil 4'te verilmiştir. Şekil 4'te görüldüğü üzere en negatif bölgeler $\mathrm{O}$ ve $\mathrm{B}$ atomları civarlarında belirlenirken, en pozitif bölgeler hidrojen atomları civarlarında görülmektedir. 3D MEP yüzey haritalarında elektrofilik baskın bölgeleri $O$ ve $B$ atomları civarlarında iken, nükleofilik atağa yatkın bölgeler ise hidrojen atomları civarlarında yoğunlaştığı görüldü.

\subsection{Geometrik optimizasyon (Geometric optimization)}

Ortorombik metaborik asit molekülünün konformasyon analizi sonucunda potansiyel enerji eğrisi üzerinde elde edilen iki minimum enerjili konformasyonlar B3LYP/6-311G++(d,p) ve HF/6-311++G(d,p) metotları ile optimize edilerek lokal minimumların en düşük enerjili olanı global minimum; C-I konformer olarak ve diğer minimum ise C-Il konformeri olarak belirlenmiştir. Her iki konformer için yapılan geometri optimizasyonu ile moleküldeki atomların uzay yapısı (bağ uzunlukları $(\AA)$, bağ açıları $\left(^{\circ}\right)$ ve dihedral açıları $\left(^{\circ}\right)$ ) teorik olarak hesaplanmıştır. Bu çalışmada, hesaplanan ve deneysel olarak belirlenen geometri parametreler [23] ile karşılaştırmalı olarak Çizelge 1'de verilmiştir. Her iki konformerin teorik bağ uzunlukları ile deneysel değerleri arasındaki uyumunu görmek için lineer regresyon analizi yapılmış ve regresyon katsayısı $\left(R^{2}\right)$ çizelgelerin sonunda verilmiştir. Molekülün $\mathrm{C}$-I konformeri için denge durumu bağ uzunlukları regresyon analizi sonucu belirlenen regresyon katsayısı sırasıyla, B3LYP/6$311++G(d, p)$ metodunda 0,9799 ve HF/6-311++G (d,p) metodunda 0,9801 ve C-II konformeri için bu değerler sırasıyla B3LYP/6-311++G(d,p) metodunda 0,9798 ve HF/6-311++G(d,p) metodunda 0,9799 olarak hesaplanmıştır. En iyi regresyon katsayısı $\left(R^{2}=0,9801\right) \mathrm{C}$-। konformeri için HF metodunda bulunmuştur. Bağ açı-

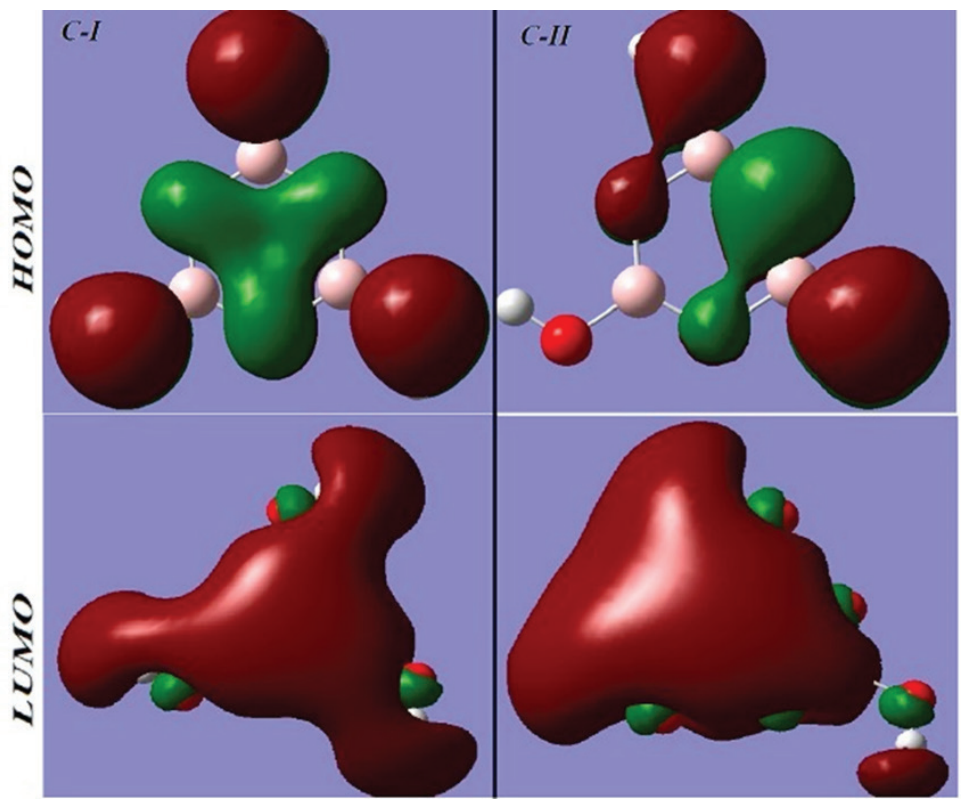

Şekil 4. C-I ve C-II konformerlerinin HOMO-LUMO çizimi B3LYP/6-311++G(d,p) (HOMO- LUMO plot of C-I and C-II conformers, B3LYP/6-311++G(d,p)). 
ları hesaplamalarında ise teorik ve deneysel bağ açıları arasında küçük sapmaların olduğu ancak maksimum sapmanın B-O-H açılarında olduğu saptandı. Bu sapmaların moleküller arası $\mathrm{O}-\mathrm{H} \cdots \cdot \mathrm{O}$ hidrojen bağları oluşumundan kaynaklandığı ve bu durumunda, kristaldeki meydana gelen moleküller arası $\mathrm{O}-\mathrm{H} \cdots \mathrm{O}$ hidrojen bağları oluşumunu kolaylaştırmaktadır. Molekülün C-I ve C-II konformerlerinin optimize edilmiş moleküler yapılar sırasıyla $\mathrm{C}_{3 \mathrm{~h}}$ ve $\mathrm{C}_{\mathrm{s}}$ simetrisine sahip olması nedeniyle; dihedral açıları $180,0^{\circ}$ ve dihedral açıları $0^{\circ}$ olarak hesaplanmış ve bu değerlerin deneysel değerlerle iyi bir uyum içinde olduğu görülmüştür. Ancak, $\Psi$ dihedral açısı C-I konformerinde 180,0 iken C-II' de $0,0^{\circ}$ 'dir. Molekülün kristal formunda yapılan deneysel yapı çalışmalarında [23] molekülün düzlemsel yapıda olduğu ve moleküldeki O-H gruplarının yönelimlerinin farklı olduğu belirlenmiştir. Bu durum ise teorik hesaplamalar sonucu elde edilen C-II konformeri ile uyumlu olduğunu gösterdi.

\subsection{HOMO-LUMO ve lineer olmayan özellikleri (HO- MO-LUMO and nonlinear property)}

Molekülün $\mathrm{C}$-I ve $\mathrm{C}$-II konformerlerinin B3LYP/6$311++G(d, p)$ ve $H F / 6-311++G(d, p)$ metodları ile hesaplanan temel durum enerjisi, dipol momenti $(\mu)$, polarizebilite $(\alpha)$, hiperpolarizebilite $(\beta)$, en yüksek dolu molekül orbital (HOMO) ve en düşük boş molekül orbital (LUMO) değerleri Çizelge 2'de verilmiştir. Molekülün C-I ve C-II konformerlerinin denge durumu elektronik enerji farkı değerleri $\left(\Delta \mathrm{E}=\mathrm{E}_{\mathrm{Cs}}-\mathrm{E}_{\mathrm{C} 3 \mathrm{~h}}\right)$ sırasıyla, B3LYP/6-311++G(d,p) metodu 0,0338 ve HF/6-311 ++ $\mathrm{G}(\mathrm{d}, \mathrm{p})$ metodu ile $0,0375 \mathrm{eV}$ olarak hesaplanmıştır. Enerji farkı değerleri molekülün C-I konformerinin C-II konformerinden daha kararlı olduğunu göstermektedir. Yapılan konformasyon analizi sonuçları, molekülün dipol moment, hiperpolarizebilite özelliklerinin molekülün konformasyonlarına önemli derecede bağlıı̆ını, yani polarizebilitenin ise etkilenmediği göstermiştir. Bu da çalışılan molekülün farklı minimumları, bazı moleküler

Çizelge 1. C-I ve C-II konformerlerinin moleküler yapı parametreleri $(6-311++G(d, p))($ molecular structure parameters for $C-I$ and C-II conformers (using 6-311++G(d,p).

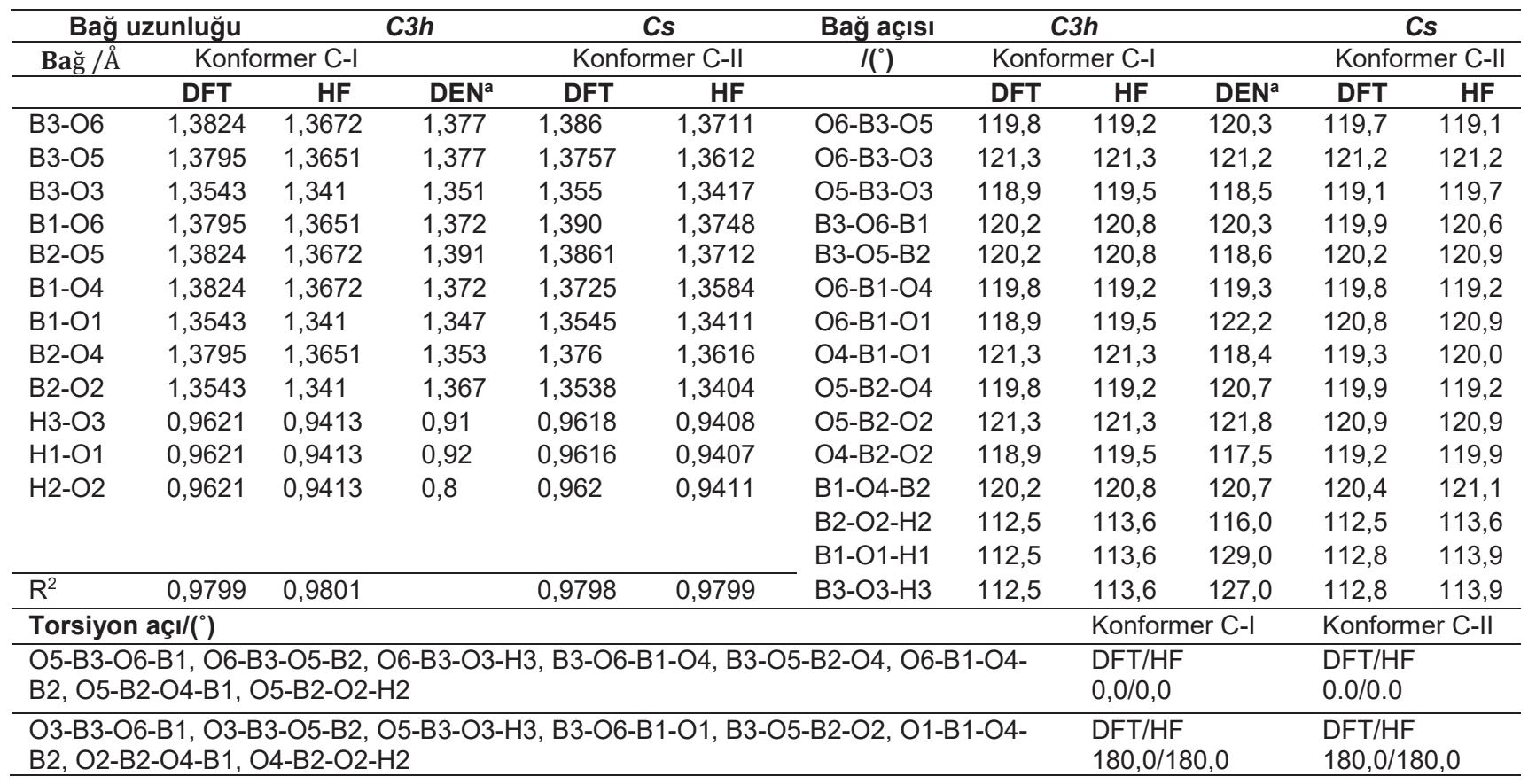

(a)Ref. 29]

Çizelge 2. C-I ve C-II konformerlerinin elektronik, HOMO, LUMO enerji, $\mu, \alpha, \beta$ ve $\Delta$ Eg değerleri (Electronic, HOMO, LUMO energy, $\mu, \alpha, \beta$ and $\Delta$ Eg values of C-I and C-II conformers).

\begin{tabular}{llllllll}
\hline \multicolumn{7}{c}{ B3LYP/6-311++G(d. p) } \\
\hline Sym. & Elektronik enerji(a.u) & $\mu(\mathrm{D})$ & $\alpha(\mathrm{a} . \mathrm{u})$ & $\beta($ a.u $)$ & $\mathrm{E}_{\text {номо }}(\mathrm{a} . \mathrm{u})$ & $\mathrm{E}_{\text {LUмо }}(\mathrm{a} . \mathrm{u})$ & $\Delta \mathrm{Eg}(\mathrm{eV})$ \\
\hline C3h & $-528,356338643$ & 0,00 & 54,36 & 1,49 & $-0,348987$ & $-0,025872$ & 8,79 \\
Cs & $-528,355096150$ & 2,95 & 54,36 & 45,11 & $-0,346798$ & $-0,029228$ & 8,64 \\
\hline \multicolumn{7}{c}{$\mathrm{HF} / 6-311++\mathrm{G}(\mathrm{d}, \mathrm{p})$} \\
\hline C3h & $-525,648822766$ & 0,00 & 44,75 & 0,31 & $-0,517622$ & 0,043209 & 15,26 \\
Cs & $-525,647446410$ & 3,07 & 44,75 & 23,72 & $-0,515640$ & 0,037839 & 15,06 \\
\hline
\end{tabular}


özelliklerinin (dipol moment ve hiperpolarizebilite gibi) farklı olduğu konformasyonlara veya izomerlere karşılık geldiğini gösterir.

Ortorombik metaborik asit molekülünün enerji aralığı daha önce teorik olarak [29] incelenmiş olup, 8,09 eV olarak belirlenmiştir. Bu çalışmada ise C-l/C-II konformeri sırasıyla B3LYP/6-311++G(d,p) 8,79/8,64 eV ve $H F / 6-311++G(d, p)$ ise 15,26/15,06 eV olarak hesaplanmıştır. B3LYP/6-311++G(d,p) yöntemi ile hesaplanan enerji aralığı değeri daha önce yapılan çaışmanın sonucu ile daha uyumlu olduğu söylenebilir [29]. Molekülün C-I ve C-Il konformerlerinin B3LYP/6$31++\mathrm{G}(\mathrm{d}, \mathrm{p})$ metodu ile elde edilen HOMO ve LUMO' ya ait üç boyutlu orbital yüzeyleri Şekil 5'te verilmiştir.

\subsection{Titreşimsel Analiz (Vibrational analysis)}

Ortorombik metaborik asit molekülünün her iki konformeri düzlemsel yapıya sahip olup, C-I konformeri $C_{3 h}$ nokta grubuna ve $\Gamma=7 A^{\prime}+3 A^{\prime \prime}+14 E^{\prime}+6 E^{\prime \prime}$ şeklinde gruplanmış toplam 30 titreşim moduna sahiptir. C-I konformerinin temel titreşim modlarından yedisi $A^{\prime}$, üçü $A$ ", on dört'ü $E$ ' ve altısı $E$ " simetri türündedir. $E$ simetri türü ikili eş enerjili (dejenere) olduğundan, E' simetri türünde on dört ve $\mathrm{E}$ " simetri türünde altı mod ikişerli dejeneredir. C-II konformeri $C_{s}$ nokta grubuna ve $\Gamma=21 A^{\prime}+9 A^{\prime \prime}$ şeklinde gruplanmış toplam 30 titreşim moduna sahiptir. Hesaplanan bu titreşim modları, potansiyel enerji dağılımı işaretlenmeleri VEDA 4f programında [40] yapılmıştır. B3LYP/6-311++G(d,p)'de hesaplanan titreşim modları 0,983 [41] ile HF/6$311++G(d, p)$ 'de ise 0,906 skala faktörü ile çarpılarak uyumlu hale getirilmiştir. Ortorombik metaborik asit molekülünün titreşim modları daha önce deneysel olarak [29,42]. (IR ve Raman) spektroskopisiyle incelenmiştir. Ancak, kristal spektrumlarda katlı ton ve frekansları temel titreşim modlarının toplamına veya farkına yakın değerlerde kombinasyon bantlarda görülmüştür. Bu çalışmada, molekülün $\mathrm{C}$-I ve $\mathrm{C}$-II konformerleri için B3LYP/6-311++G(d,p) ve HF/6-311++G(d,p) yöntemi ile hesaplanan FT-IR spektrumları sırasıyla Şekil 6 (a) ve (b)'de ve Raman spektrumları Şekil 7 (a) ve (b)'de verilmiştir. Molekülün her iki konformerinin her iki yöntemi ile elde edilen titreşim modları ise Çizelge 3 ve 4'te verilmiştir. Ayrıca, molekülün konformerlerinin farklı nokta grubuna sahip olmasına rağmen her iki hesaplama yöntemde hesaplanan titreşim modlarının hemen hemen aynı eğilimde oldukları Çizelge 3 ve 4'te görülmektedir. Ortorombik metaborik asit molekülünün $\mathrm{O}-\mathrm{H}$ gruplarının gerilme titreşimleri; C-I konformeri için B3LYP/6-311++G(d,p)'de 3711, 3710, $3710 \mathrm{~cm}^{-1}$

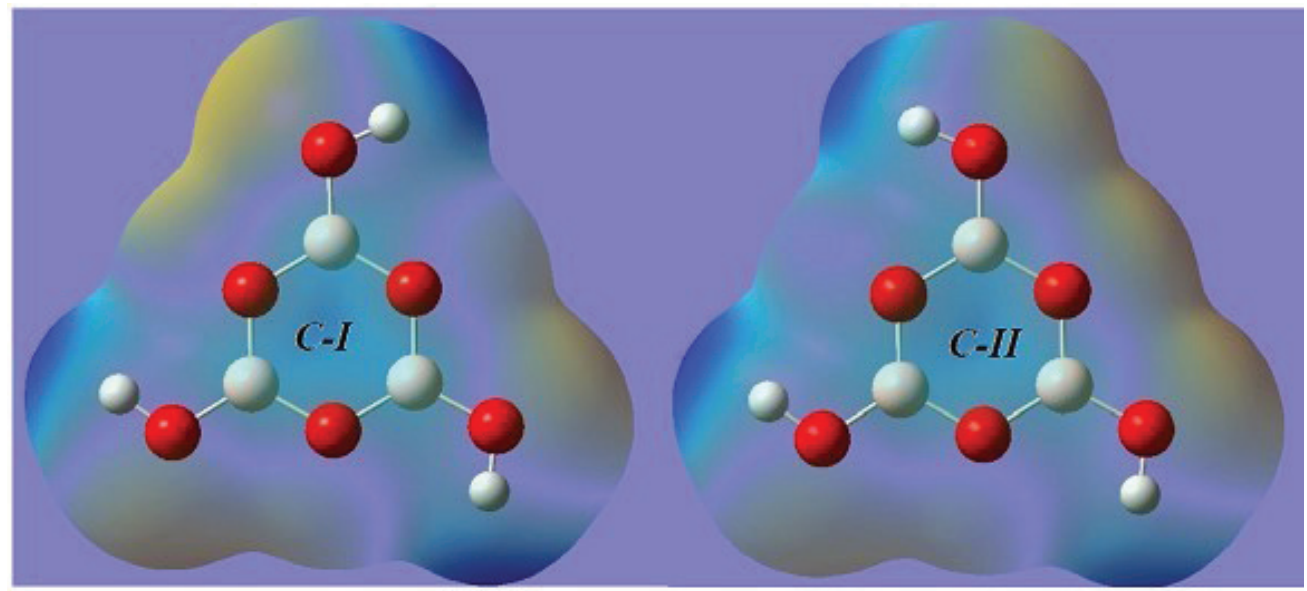

Şekil 5. C-I ve C-II konformerlerinin MEP yüzeyi (MEP surfaces of C-I and C-II conformers).
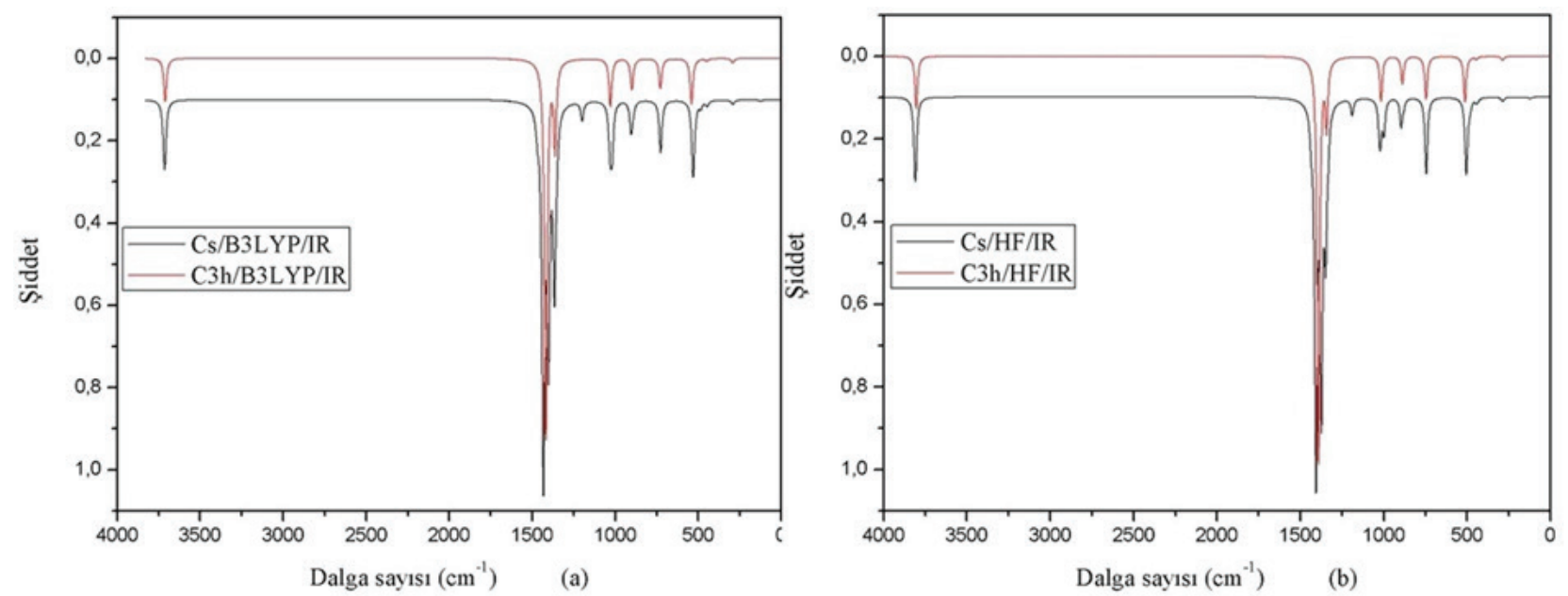

Şekil 6. C-I ve C-II konformerlerinin hesaplanmış FT-IR spektrumları (Calculated FT-IR spectra of C-I and C-II conformers). 


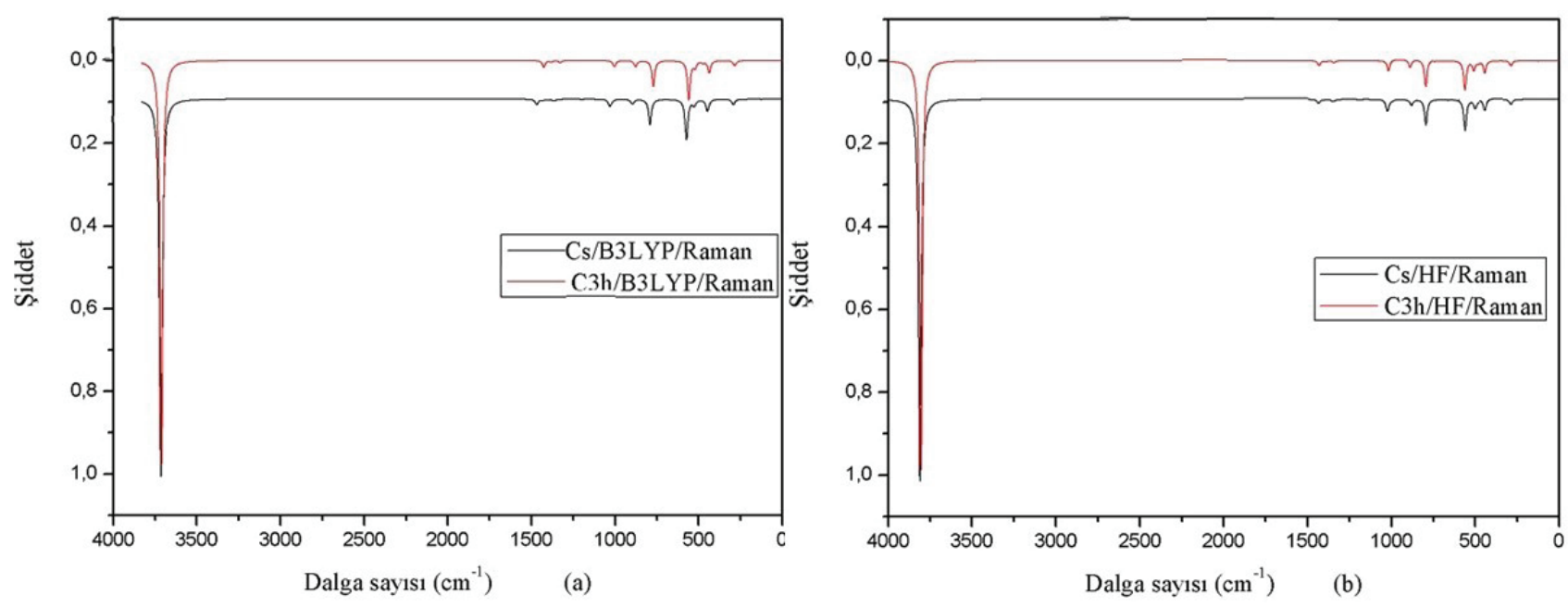

Şekil 7. C-I ve C-II konformerlerinin hesaplanmış FT-IR spektrumları (calculated FT-Raman spectra of C-I and C-II conformers).

ve HF/6-311++G(d,p)'de 3803, 3802, $3802 \mathrm{~cm}^{-1}$, C-II konformeri için sırasıyal $3716,3713,3710 \mathrm{~cm}^{-1}$ ve $3811,3808,3805 \mathrm{~cm}^{-1}$ olarak hesaplanmıştır. Bu gerilim titreşimleri deneysel olarak sırasıyla 3375,3280 , $3200 \mathrm{~cm}^{-1}$ [29] ve $33653295,3195 \mathrm{~cm}^{-1}$ [42] bantlarında gözlenmiştir. Daha önceki yaptığımız çalışmada boronik asit O-H grubunun gerilme titreşimleri 3687$3596 \mathrm{~cm}^{-1}$ gözlendi [43]. Deneysel ve teorik değerler karşılaştırılığında; deneysel çalışmaların molekül içi ve moleküller arası oluşan (hidrojen bağları gibi) etkileşmelerin olduğu kristal (katı) fazında teorik çalışmalar ise izole durumundaki molekülün gaz fazında yapılmaktadır. Molekülde hidrojen bağları yapan atomların gerilim titreşimlerinin daha büyük dalga boylarına kaydığı saptanmıştır. Deneysel olarak 1473 [27] ve 1475 [39] B-O gerilim titreşimi teorik olarak C-I konformeri için B3LYP/6-311++G(d,p)/HF/6$311++G(d, p)$ 'da, $1462 / 1429 \mathrm{~cm}^{-1}$ ve C-Il konformeri için sırasıyla $1429 / 1433 \mathrm{~cm}^{-1}$ olarak hesaplanmıştır. B3LYP/6-311++G(d,p) yöntemi ile hesaplanan B-O gerilim titreşiminin deneysel değerle daha uyumlu olduğu görülmüştür.

Çizelge 3. C-I konformerinin 6-311++G(d,p) 'de hesaplanmış dalga sayıları $\left(\mathrm{cm}^{-1}\right)$ (Calculated wave numbers of $C-I$ conformer at 6-311 ++ G (d, p) $\left.\left(\mathrm{cm}^{-1}\right)\right)$.

\begin{tabular}{|c|c|c|c|c|}
\hline \multirow{2}{*}{$\begin{array}{c}\text { Mod } \\
\text { no }\end{array}$} & \multicolumn{2}{|r|}{ B3LYP } & HF & \\
\hline & Sym. & Skalalı & Skalalı & İşaretlemeler v;gerilme $\delta$;düzlem içi bükülme т;düzlem dışı bükülme \\
\hline $\mathrm{v} 1$ & $A^{\prime}$ & 3711 & 3803 & $v \mathrm{O} 1 \mathrm{H}(93)$ \\
\hline v2 & $E^{\prime}$ & 3710 & 3802 & $v \mathrm{O} 2 \mathrm{H}(93)$ \\
\hline v3 & $E^{\prime}$ & 3710 & 3802 & $v \mathrm{O} 3 \mathrm{H}(100$ \\
\hline v4 & $A^{\prime}$ & 1462 & 1429 & $v \mathrm{O} 3 \mathrm{~B} 3(45) v \mathrm{O} 4 \mathrm{~B} 2(15) \mathrm{\delta B} 1 \mathrm{O} 4 \mathrm{~B} 2(10)$ \\
\hline v5 & $E^{\prime}$ & 1416 & 1390 & $v \mathrm{O} 6 \mathrm{~B} 1(50) v \mathrm{O} 2 \mathrm{~B} 2(29) v \mathrm{O} 5 \mathrm{~B} 3(10) v \mathrm{O} 5 \mathrm{~B} 2(11)$ \\
\hline v6 & $E^{\prime}$ & 1416 & 1390 & $v \mathrm{O} 4 \mathrm{~B} 1(30)$ \\
\hline v7 & $E^{\prime}$ & 1361 & 1341 & $v \mathrm{O} 6 \mathrm{~B} 1(12) v \mathrm{O} 2 \mathrm{~B} 2(12) v \mathrm{O} 1 \mathrm{~B} 1(11) \delta \mathrm{O} 6 \mathrm{~B} 1 \mathrm{O} 4(17)$ \\
\hline v8 & $E^{\prime}$ & 1361 & 1341 & $v \mathrm{O} 1 \mathrm{~B} 1(14) v \mathrm{O} 5 \mathrm{~B} 3(42) v \mathrm{O} 4 \mathrm{~B} 1(20)$ \\
\hline v9 & $A^{\prime}$ & 1202 & 1193 & $v \mathrm{O}$ В2(59) סНО2B2(11) \\
\hline v10 & $A^{\prime}$ & 1035 & 1025 & $v$ O5B2(11) סНО2B2(79) \\
\hline v11 & $E^{\prime}$ & 1029 & 1016 & $v \mathrm{O} 2 \mathrm{~B} 2(20) v \mathrm{O} 1 \mathrm{~B} 1(21) \delta \mathrm{HO} 3 \mathrm{~B} 2(45)$ \\
\hline v12 & $E^{\prime}$ & 1029 & 1016 & $v \mathrm{O} 4 \mathrm{~B} 1(17) \delta \mathrm{HO} 1 \mathrm{~B} 1(46)$ \\
\hline v13 & $E^{\prime}$ & 899 & 887 & $v \mathrm{O} 6 \mathrm{~B} 1(11) v \mathrm{O} 5 \mathrm{~B} 3(21) \delta \mathrm{O} 6 \mathrm{~B} 1 \mathrm{O} 4(11) \delta \mathrm{B} 1 \mathrm{O} 4 \mathrm{~B} 2(10)$ \\
\hline v14 & $E^{\prime}$ & 899 & 887 & $v \mathrm{O} 1 \mathrm{~B} 1(27) \delta \mathrm{HO} 3 \mathrm{~B} 2(32) \delta \mathrm{HO} 1 \mathrm{~B} 1(33)$ \\
\hline v15 & $A^{\prime}$ & 791 & 794 & 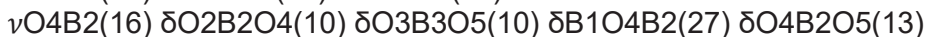 \\
\hline v16 & A" & 728 & 746 & тВ104B2O5(21) тO1O6O4B1(76) \\
\hline v17 & E" & 662 & 665 & TO2O5O4B2(88) \\
\hline v18 & E" & 662 & 665 & тO30605B3(85) \\
\hline v19 & $A^{\prime}$ & 573 & 560 & $v \mathrm{O} 3 \mathrm{~B} 3(28) v \mathrm{O} 4 \mathrm{~B} 2(32) \delta \mathrm{B} 1 \mathrm{O} 4 \mathrm{~B} 2(23) \delta \mathrm{O} 4 \mathrm{~B} 2 \mathrm{O} 5(12)$ \\
\hline v20 & A" & 540 & 512 & $\mathrm{THO} 2 \mathrm{~B} 2 \mathrm{O} 5(64)$ \\
\hline v21 & E" & 535 & 508 & тНО3В2O6(93) \\
\hline v22 & E" & 535 & 508 & тHO1B106(96) \\
\hline v23 & $A^{\prime}$ & 485 & 481 & бО3В305(77) \\
\hline v24 & $E^{\prime}$ & 447 & 443 & 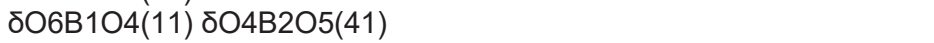 \\
\hline v25 & $E^{\prime}$ & 447 & 443 & бВ3О5В2(72) бВ104В2(12) \\
\hline v26 & $E^{\prime}$ & 292 & 287 & $\delta \mathrm{O} 6 \mathrm{~B} 104(35) \delta \mathrm{O} 2 \mathrm{~B} 2 \mathrm{O} 4(40)$ \\
\hline v27 & $E^{\prime}$ & 292 & 287 & бO1B104(57) \\
\hline v28 & A" & 129 & 125 & тВ104B2O5(77) тO10604B1(23) \\
\hline v29 & E" & 124 & 122 & тВ3O5B2O4(95) \\
\hline v30 & E" & 124 & 122 & тO6B104B2(96) \\
\hline
\end{tabular}


Çizelge 4. C-II konformerinin 6-311++G(d,p) 'de hesaplanmış dalga sayıları $\left(\mathrm{cm}^{-1}\right)$ (Calculated wave numbers of $C-I l$ conformer at $\left.6-311++\mathrm{G}(\mathrm{d}, \mathrm{p})\left(\mathrm{cm}^{-1}\right)\right)$.

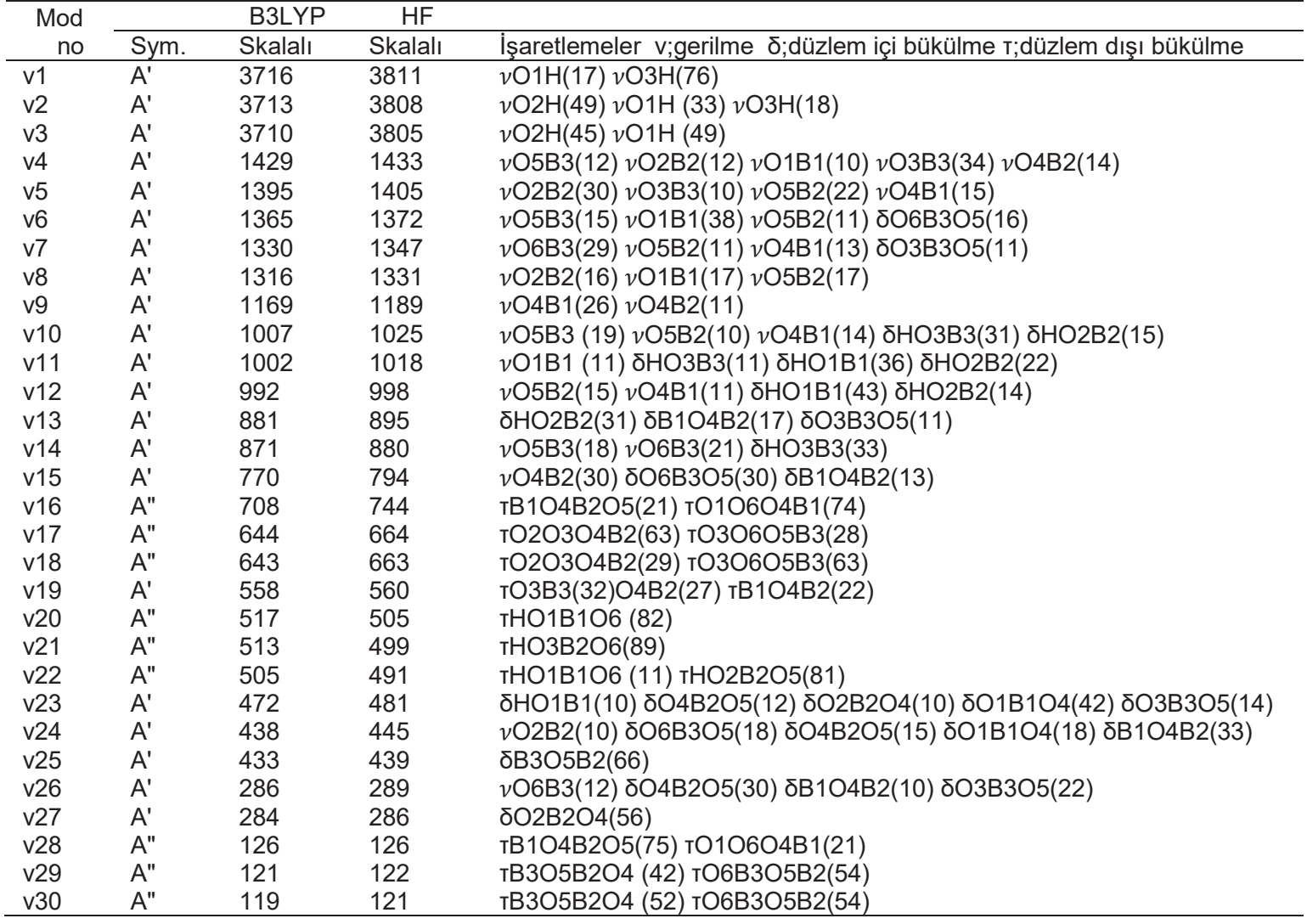

\section{Sonuçlar (Conclusions)}

Ortorombik metaborik asit molekülünün konformasyon analizi $\varphi(\mathrm{B} 1-\mathrm{O} 1-\mathrm{H})$ bağ açısı ve dihedral açının $\Psi$ (O4-B1-O1-H) fonksiyonu olarak yapılmış ve molekülün iki kararlı durum konformerleri (C-I ve C-II) belirlenmiştir. Bu konformerlerin yapısal parametreleri, dipol moment $(\mu)$, polarizebilite $(\alpha)$, hiperpolarizebilite $(\beta)$, en yüksek dolu molekül orbital enerjileri (HOMO), en düşük boş molekül orbital enerjileri (LUMO) ve temel titreşim modları değerleri DFT/B3LYP ve HF metodları ve $6-311++G(d, p)$ taban seti kullanılarak hesaplanmıştır. Ayrıca, HOMO ve LUMO enerjileri kullanılarak molekülün enerji aralığı belirlenmiştir. Molekülün C-I ve C-II konformerlerinin titreşim frekanslarının da ihmal edilebilir dercede kaymalar olduğu gözlemlenmiştir. Fakat her iki durumda da titreşim frekanslarında negatif değer görülmemiştir. Konformasyon analizi sonuçları, molekülün dipol moment, hiperpolarizebilite özelliklerinin molekülün konformasyonlarına önemli derecede bağlılığını, polarizebilitenin ise etkilenmediğini göstermiştir. Molekülün konformerleri arasında enerjinin küçük olması molekülün her iki konformerde bulunma ihtimalinin hemen hemen artıracağı gibi molekülün bazı yapısal ve elektronik özelliklerinin etkilendiği görülmüştür.

\section{Kaynaklar (References)}

[1] Kur K., Kowalska-Baron A., Miller E., The DFT study on the electronic structure of boronic acid derivatives and its esters with fructose, Biotechnol. Food. Sci., 82 (1), 29-39, 2018.
[2] Neupane L. N., Lohani C. R., Kim J., Lee K. H., A dual role of phenylboronic acid as a receptor for carbohydrates as well as a quencher for neighboring pyrene fluorophore, Tetrahedron, 69, 11057-11063, 2013.

[3] Duydu Y., Başaran N., Bolt H.M., Exposure assessment of boron in Bandırma boric acid production plant, J. Trace. Elem. Med. Biol., 26,161-4, 2012.

[4] Turkez H., Geyikoglu F., Tatar A., Keles M. S., Kaplan I., The effects of some boron compounds against heavy metal toxicity in human blood, Exp. Toxicol. Pathol., 64, 93-101, 2012.

[5] Bezerra da Silva M., dos Santos R. C. R., da Cunha A. M., Valentini A., Pessoa O. D. L., Caetano E. W. S, Freire V. N., Structural, electronic, and optical properties of bulk boric acid 2a and 3t polymorphs: Experiment and density functional theory calculations, Cryst. Growth. Des., 16, 11, 6631-6640, 2016.

[6] Demirtaş A., Bor bileşikleri ve tarimda kullanimi, Atatürk Üniv. Ziraat Fak. Derg. 37 (1), 111-115, 2006.

[7] Pizzorno L., Nothing boring about boron, Integr. Med. (Encinitas), 14, 35-48, 2015.

[8] Groziak M. P., Boron therapeutics on the horizon, Am. J. Ther., 8, 321-328, 2001.

[9] Kingma $H_{\text {. }}$, The pharmacology and toxicology of boron compounds, Can. Med. Assoc. J., 78, 620-622, 1958.

[10] Das B. C., Thapa P., Karki R., Schinke C., Das S., Kambhampati S., Banerjee S. K., Van Veldhuizen P., Verma A., Weiss L. M., Evans T., Boron chemicals in diagnosis and therapeutics, Future Med. Chem., 6, 653-676, 2013. 
[11] Del Rosso J. Q., Plattner J. J., From the test tube to the treatment room: fundamentals of boron-containing compounds and their relevance to dermatology, J. Clin. Aesthet. Dermatol., 2, 13-21, 2014.

[12] Benelli G., Mehlhorn H., Declining malaria, rising of dengue and zika virus: Insights for Mosquito Vector Control, Parasitol. Res., 115, 1747-1754, 2016.

[13] Murray N. E. A., Quam, M. B., Wilder-Smith A., Epidemiology of dengue: Past present and future prospects, Clin. Epidemiol., 5, 299-309, 2013.

[14] Didier Musso D. J. G., Zika Virus, Clin. Microbiol. Rev., 29, 487-524, 2016

[15] Bhami L. C., Das S. S. M., Boric acid ovicidal trap for the management of aedes species, J. Vector Borne Dis., 52,147-152, 2015.

[16] Qualls W. A., Müller, G. C., Traore S. F., Traore M. M., Arheart K. L., Doumbia S., Schlein Y., Kravchenko V. D., Xue R. D., Beier J. C., Indoor use of attractive toxic sugar bait (ATSB) to effectively control malaria vectors in Mali, West Africa. Malar. J., 14, 301, 1-8, 2015.

[17] Yılmaz Aydın, D., Gürü, M., Ayar, B., Çakanyıldırım, Ç., Bor bileşiklerinin alev geciktirici ve yüksek sıcaklığa dayanıklı pigment olarak uygulanabilirliği, Boron 1 (1), 33 - 39, 2016.

[18] Delpierre S., Willocq B., De Winter J., Dubois P., Gerbaux P., Raquez J. M., Dynamic Iminoboronate-based boroxine chemistry for the design of ambient humiditysensitive self-healing polymers, Chem. A Eur. J., 23, 6730-6735, 2017.

[19] Kalemos A., The nature of the chemical bond in borazine $(\mathrm{B} 3 \mathrm{~N} 3 \mathrm{H} 6)$, boroxine $(\mathrm{B} 3 \mathrm{O} 3 \mathrm{H} 3)$, carborazine (B2N2C2H6), and related species, Int. J. Quantum Chem., 118, 1-8, 2018.

[20] Kracek F. C., Morey G. W., Merwin H. E., The system water-Boron oxide, Amer. J, Sci., 81, 229-234, 1938.

[21] Kilday M. V., Prosen E. J., Heats of solution, transition, and formation of three crystalline forms of metaboric acid, journal of research of the national bureau of standards-A, Phys. Chem., 68A, 1, 127-144, 1964.

[22] Töre İ., Ay N., Amorf boron oksit eldesi ve Karekterizasyonu, II. Uluslararası bor sempozyumu, EskişehirTürkiye, 23-25 Eylül, 2004.

[23] Peters C.R., Mılberg M.E., The refined strucrure of orthorhombic metaboric acid, Acts Cryst.,17, 229-234, 1964.

[24] Zacharlasen W. H., The crystal structure of monoclinic metaboric acid, Acta Cryst., 16, 385-389, 1963.

[25] Zacharlasen W. H., The crystal structure of cubic metaboric acid, Acta Cryst., 16, 380-383, 1963.

[26] Bertoluzza A., M, P., Battaglıa M. A., Bonora S., Infrared and raman spectra of orthorhombic, monoclinic and cubic metaboric acid and their relation to the "strength" of the hydrogen bond present, J. Mol. Struct., 64 123136, 1980.

[27] Broadhead P., Newman, A., The vibrational spectra of orthoboric acid and its thermal deconposition products, J. Mol. Struct., 19, 157-171 1971.

[28] Sürdem, S., Synthesis and characterization of trimethoxy boroxine, Boron, 4 (3), 148-152, 2019.
[29] Bezerra da Silva M., Da Cunha M., Santos R. C. R., Valentini A., Caetano E. W. S., Freireab V. N., Changing the gap type of solid state boric acid by heating: $A$ dispersion-corrected density functionalstudy of $\alpha-, \beta-$, and $\mathrm{y}$-metaboric acid polymorphs, New J. Chem., 41, 15533-15544, 2017.

[30] Dennington R., Keith T., Millam J., Semichem Inc., Gauss View, Version 5, Shawnee Mission KS, 2009.

[31] Frisch M. J., Trucks G. W., Schlegel H. B., Scuseria G. E., Robb M. A., Cheeseman J. R., Scalmani G., et al., Gaussian Inc., (Wallingford, CT), 2010.

[32] Becke A. D., Density-functional exchange-energy approximation with correct asymptotic behaviour, Phys. Rev. A, 38 (6), 3098-310, 1988.

[33] Becke A. D., Density-functional thermochemistry 3. the role of exact exchange, J. Chem. Phys., 98 (7), 56485652, 1993.

[34] Lee C. T., Yang W. T., Parr R. G., Development of the colle-salvetti correlation-energy formula into a functional of the electron density, Phys. Rev., B, 37, 785789,1988 .

[35] Frisch M. J., Pople J. A., Binkley J. S., Selfconsistent molecular orbital methods 25, Supplementary functions for Gaussian basis sets, J. Chem. Phys., 80, 3265-3269, 1984.

[36] Driss M., Benhalima N., Megrouss Y., Rachida R., Chouaih A., Hamzaoui F., Theoretical and experimental electrostatic potential around the m-nitrophenol molecule, Molecules, 20, 4042-4054, 2015.

[37] Haress N. G., El-Emam A., Al-Deab O. A., Panicker C. Y., Al-Saadi A., Van Alsenols C., Ahmad War J., Vibrational spectroscopic and molecular dockings tudy of 2-benzylsulfanyl-4-[(4-methylphenyl)-sulfanyl]6-pentylpyrimidine-5-carbonitrile, a potential chemo the rapeutic agent, Spectrochim. Acta A, 137, 569-580, 2015.

[38] Mulliken R. S., Electronic population analysis on LCAO-MO molecular wave functions, J. Chem. Phys., 23 (10), 1833-1840, 1955.

[39] Politzer P., Murray J. S., Concha M. C., The complementary roles of molecular surface electrostatic potentials and average local ionization energies with respect to electrophilic processes, Int. J. Quantum Chem., 88 (1), 19-27, 2002.

[40] Jomroz M. H., Vibrational Energy distribution Analysis VEDA4 (Warsaw), 2004

[41] Sundaraganesana N., Ilakiamania S., Saleema H., Wojciechowskib P. M., Michalskab D., FT-raman and FT-IR spectra, vibrational assignments and density functional studies of 5-bromo-2-nitropyridine, Spec. Acta Part A, 61, 2995-3001, 2005.

[42] Parsons J. L., Vibrational spectra of orthorhombic metaboric acid, J. Chem. Phys., 33, 1860-1866, 1960.

[43] Uğurlu G., 2-metoksipiridin-3-boronik asitin lineer olmayan özellikleri, konformasyonel, titreșimsel ve elektronik yapısı üzerine substitüent etkisinin kuantum mekanik metodlar ile araştırılması, Erzincan University Journal of Science and Technology. 12(1), 14-24, 2019. 